\title{
Psychosocial stress predicts abnormal glucose metabolism: The Australian Diabetes Obesity and Lifestyle (AusDiab) Study
}

\section{Emily D. Williams ${ }^{1,2}$; Dianna J. Magliano ${ }^{3}$; Robyn J. Tapp ${ }^{4}$; Brian F. Oldenburg ${ }^{1}$; Jonathan E. Shaw ${ }^{3}$}

\footnotetext{
${ }^{1}$ School of Public Health and Preventive Medicine, Monash University, Australia.

${ }^{2}$ International Centre for Circulatory Health, National Heart and Lung Institute, St Mary’s Hospital and Imperial College London, UK

${ }^{3}$ Baker IDI Heart and Diabetes Institute, Melbourne, Australia.

${ }^{4}$ National Vision Research Institute, Australian College of Optometry, Melbourne, Australia.
}

Corresponding address: Emily Williams, School of Public Health and Preventive Medicine, Monash University, Alfred Hospital, 89 Commercial Road, Melbourne, VIC 3004, Australia, +61 (0) 399030255 (tel), emily.d.williams@ monash.edu (email).

\section{Acknowledgements}

EDW is supported by a Diabetes UK Fellowship (09/0003833). DJM is supported by the Victorian Cancer Agency Fellowship for 2011-2012. RJT is supported by a Senior Research Lions Foundation fellowship. JES is supported by a National Health and Medical Research Council Fellowship (586623). The AusDiab study, co-coordinated by the Baker IDI Heart and Diabetes Institute, gratefully acknowledges the generous support given by: National Health and Medical Research Council (NHMRC grant 233200), Australian Government Department of Health and Ageing. Abbott Australasia Pty Ltd, Alphapharm Pty Ltd, AstraZeneca, Bristol-Myers Squibb, City Health Centre-Diabetes Service-Canberra, 
Department of Health and Community Services - Northern Territory, Department of Health and Human Services - Tasmania, Department of Health - New South Wales, Department of Health - Western Australia, Department of Health - South Australia, Department of Human Services - Victoria, Diabetes Australia, Diabetes Australia Northern Territory, Eli Lilly Australia, Estate of the Late Edward Wilson, GlaxoSmithKline, Jack Brockhoff Foundation, Janssen-Cilag, Kidney Health Australia, Marian \& FH Flack Trust, Menzies Research Institute, Merck Sharp \& Dohme, Novartis Pharmaceuticals, Novo Nordisk Pharmaceuticals, Pfizer Pty Ltd, Pratt Foundation, Queensland Health, Roche Diagnostics Australia, Royal Prince Alfred Hospital, Sydney, Sanofi Aventis, Sanofi Synthelabo. The AusDiab study was also supported in part by the Victorian Government's OIS Program. For their invaluable contribution to the AusDiab set-up and field activities, we are enormously grateful to A. Allman, B. Atkins, S. Bennett, A. Bonney, S. Chadban, M. de Courten, M. Dalton, D. Dunstan, T. Dwyer, H. Jahangir, D. Jolley, D. McCarty, A. Meehan, N. Meinig, S. Murray, K. O’Dea, K. Polkinghorne, P. Phillips, C. Reid, A. Stewart, R. Tapp, H. Taylor, T. Whalen, F. Wilson and P Zimmet. 


\title{
Psychosocial stress predicts abnormal glucose metabolism: The Australian Diabetes Obesity and Lifestyle (AusDiab) Study
}

\begin{abstract}
Background: The evidence supporting a relationship between stress and diabetes has been inconsistent.

Purpose: This study examined the effects of stress on abnormal glucose metabolism, using a population-based sample of 3759 , with normoglycemia at baseline, from the Australian Diabetes, Obesity and Lifestyle (AusDiab) study.

Methods: Perceived stress and stressful life events were measured at baseline, with health behavior and anthropometric information also collected. Oral glucose tolerance tests were undertaken at baseline and five-year follow-up. The primary outcome was the development of abnormal glucose metabolism (impaired fasting glucose, impaired glucose tolerance, and type 2 diabetes), according to WHO 1999 criteria.
\end{abstract}

Results: Perceived stress predicted incident abnormal glucose metabolism in women but not men, after multivariate adjustment. Life events showed an inconsistent relationship with abnormal glucose metabolism.

Conclusions: Perceived stress predicted abnormal glucose metabolism in women. Healthcare professionals should consider psychosocial adversity when assessing risk factor profiles for the development of diabetes.

Keywords Type 2 diabetes; Stress; Risk factors: Prevention; Health behaviors. 
The prevalence of diabetes is rising rapidly worldwide, with projections estimating that there will be up to 439 million people globally living with diabetes by 2030 (1). Considerable research has confirmed biological and behavioral variables as risk factors for the development of type 2 diabetes (2). However, the role of psychosocial risk factors, including psychological characteristics, social relationships and stressors, in the development of diabetes has received much less attention. Despite the established link between psychosocial adversity and the development of coronary heart disease (CHD) (3-4), and the fact that there is significant overlap in risk factors and underlying pathology between diabetes and CHD (5), the role of psychosocial risk factors remains under-researched in the diabetes field.

The previous work in this area has shown that psychological characteristics, such as depression and psychological distress (anxiety and depression), independently predict the development of abnormal glucose metabolism (6-8). A meta-analyses by Knol et al. indicated that adults with depression had a 37\% increased risk of developing diabetes, compared with people with no depression (7). Limited research has also explored the role of social relationships on diabetes risk, with one Swedish study showing increased odds of up to three times the risk of future diabetes among women with weak emotional support compared with those with strong support, a relationship that was not observed in men (9).

Only a small number of studies have explored the role of psychosocial stress on the development of diabetes. Stress can be measured as the actual exposure to events assumed to be stressful, or as an individual's interpretation and perception of stressors (10), and can be assessed across all contexts of life. Work from the Coronary Artery Risk Development in Young Adults (CARDIA) study demonstrated a prospective effect of financial strain on impaired fasting glucose over a 20 year follow-up period (11). To date, however, the majority of the research in this area has explored the relationship between work-related stress 
and diabetes $(9,12-18)$; many studies have used select populations and the findings have been contradictory $(13,17,19-20)$. Two papers from the Whitehall II study, for example, report inconsistent findings across genders; using a sample of British civil servants, the first paper found a relationship between one measure of work stress, effort-reward imbalance (a model based on the importance of the reciprocal balance between the effort expended at work and the intrinsic and extrinsic rewards received (21)) and diabetes incidence in men, but not in women (17). In contrast, a later article demonstrates another measure of work stress, isostrain (the combination of high demands, low control and social isolation (22)) as a predictor of diabetes incidence in women, but not men (13). A meta-analysis of the work stressdiabetes literature failed to show statistically significant relationships between any individual aspect of work-related stress and risk of diabetes (23).

These Whitehall II studies $(13,17)$ and findings from the cardiovascular literature highlight the potential gender differences in the impact of stress on health, with a number of explanations proposed to explain these variations across gender. Toker et al found a differential inflammatory response to stress between men and women (24); in women, burnout and anxiety were associated with C-reactive protein and fibrinogen, while in men, only depression was related to these inflammatory markers. Other studies have demonstrated a similar but heightened stress response with greater impact on cardiovascular risk factors among women compared with men, as a result of increased sensitivity to stress (25). In addition, different types of stress appear to affect women and men, with women more likely to report stress associated with their social network and men with their work environment (26).

Although studies have reported an association between more general measures of stress and diabetes risk, this research is limited by its use of cross-sectional data (27), crude measures of stress and self-reported diabetes (19), as well as small samples (28). 
Furthermore, the findings from such studies are conflicting $(17,27,29)$. It is clear that more prospective work is needed to overcome the methodological inconsistencies of previous studies (23) and understand the impact of different types of stressors on the development of diabetes.

These analyses test the hypothesis that psychosocial stressors are associated with increased development of abnormal glucose metabolism in a national, population-based sample from Australia, using objective markers of glucose tolerance. In addition, we aimed to compare the effects of a marker of perceived stress, with a measure of 'objective' stress experience, stressful life events, to explore possible differential effects on abnormal glucose metabolism according to stress type in men and women.

\section{Methods}

\section{Study Population}

The Australian Diabetes Obesity and Lifestyle study (AusDiab) baseline methods and response rates have been described in detail elsewhere (30). The study involved a national, population-based survey of adults aged 25 years in 1999-2000, using a stratified cluster design with sample drawn from 42 randomly selected census collector districts across Australia. Information was collected using a brief household interview, followed by a biomedical examination. Of the adults eligible to participate, $70 \%$ completed the household interview, and 55\% $(\mathrm{n}=11,247)$ of these completed the baseline biomedical examination (30). All living eligible participants were invited to attend a follow-up assessment in 20042005 (ineligibility was based on refusal for further contact, death, moving abroad, living in a nursing facility classified for high care, or terminal illness). Among the eligible participants, $59 \%$ returned for the five-year follow-up where baseline assessment was repeated. Compared with non-responders, attendees were significantly less likely to be hypertensive, to 
have a lower level of education attainment, or to be smokers and to have lower 2-hour plasma glucose, glycated hemoglobin, and smaller waist circumferences at baseline. Perceived stress did not vary between attendees and non-responders, although stressful life events were higher among the non-responders.

\section{Baseline measurements}

At baseline (and follow-up), the physical examination included blood samples, anthropometric measurements, and questionnaires. All participants, except for those currently receiving treatment for diabetes or who were pregnant, underwent a standard 75-g oral glucose tolerance test (30). Details on the glucose and lipid assays used between studies can be found in detail elsewhere (2). Blood pressure was measured using Dinamap or a standard mercury sphygmomanometer, in a seated position after participants had rested for at least 5 minutes. Three readings were taken at 1-minute intervals, with the mean of the first two readings recorded. If the difference between the three readings was greater than 10 $\mathrm{mmHg}$, the mean of the two closest measurements was used. Height, weight and waist circumference were measured by the trained research staff.

Individual-level socioeconomic position data (education and total household income) were collected by an interviewer-administered questionnaire at baseline, as was information on smoking, leisure-time physical activity, and family history of diabetes. Following responses to the question enquiring about the highest educational qualifications received, education was classified into four categories: 1) up to secondary school education; 2) trade/technical certificates; 3) associate degree, undergraduate diplomas, nursing/teaching qualifications; and 4) bachelor degree, post-graduate qualifications. The Active Australia questionnaire measured total leisure-time physical activity in minutes reported for the previous week (31). Smoking history (current smoker, past smoker, or never smoked) was 
collected by questionnaire, previously validated in Australian adults (32). Daily alcohol consumption was assessed with the self-administered Anti-Cancer Council of Victoria food frequency questionnaire (33). Sedentary behavior was measured using self-reported television viewing as used previously (34); this determined the total time spent watching television or videos in the preceding week. As a result of the large number of zero values reported for alcohol and sedentary behavior variables, constants were added to all values and these variables were logarithmically transformed.

Psychosocial adversity was measured at baseline by two scales. Perceived stress was measured using the Perceived Stress Questionnaire (35), which comprises 30 items assessing perceptions of stress (see table 1 for list of items) over the past year. Respondents were asked to choose their level of agreement with each statement, ranging from almost never to usually on a four-point Likert scale. The Perceived Stress Questionnaire index was derived from the raw scores, and was calculated to range from 0 to 1 (35) (higher scores reflecting elevated perceived stress); these were then categorized into quartiles. The internal consistency for this scale was excellent (Cronbach's $\alpha=0.93$ ). A previous validation study of the Perceived Stress Questionnaire identified four factors of worries, tension, joy and demands, the first three of which represent internal reactions to stress, with demands related to external stressors (36). These four components were categorized into quartiles and explored in sensitivity analyses in this study.

The 'objective' experience of stress was assessed with a life events scale covering the preceding 12 months (37). This scale lists 13 adverse, (potentially) stressful life events (such as marriage breakdown, financial hardship, and death of a family member) (see table 1); experience of each stressor was recorded and summed, and total scores were categorized into four groups $(0,1,2,3$ or more events). This scale demonstrated satisfactory internal consistency (Cronbach's $\alpha=0.61)$. 


\section{Follow-up measurement of abnormal glucose metabolism}

The categories of impaired fasting glucose (fasting plasma glucose $6.1-6.9 \mathrm{mmol} / \mathrm{l}$ and 2-hour plasma glucose $<7.8 \mathrm{mmol} / \mathrm{l}$ ), impaired glucose tolerance (fasting plasma glucose $<7.0 \mathrm{mmol} / \mathrm{l}$ and 2-hour plasma glucose $\geq 7.8 \mathrm{mmol} / 1$ but $<11.1 \mathrm{mmol} / \mathrm{l}$ ), and diabetes (fasting plasma glucose $\geq 7.0 \mathrm{mmol} / 1$ or 2 -hour plasma glucose $\geq 11.1 \mathrm{mmol} / 1$ or current treatment with insulin or oral hypoglycemic agents) at five-year follow-up had previously been classified according to the 1999 WHO diabetes classification (38). These three categories were pooled to comprise the outcome category of incident abnormal glucose metabolism.

\section{Statistical analysis}

Comparisons of baseline characteristics between categories of glucose metabolism were conducted using analyses of covariance, logistic regression and Mann-Whitney tests as appropriate, adjusting for age differences, and stratified by gender. The analyses examined the role of baseline perceived stress and stressful life events on the development of abnormal glucose metabolism at follow-up, using logistic regression analyses. Only people with complete follow-up data and normoglycemia at baseline were included ( $\mathrm{n}=3,759$; of the 6290 people who provided glucose tolerance data at follow-up, 1575 with baseline abnormal glucose metabolism were excluded, as well as an additional 956 with missing data across all variables of interest). We tested the impact of these missing data, by comparing the relationship between stress and abnormal glucose metabolism (adjusted only for age and gender) in all participants (including those with missing data for other covariates used in full models, $n=4444$ ) with only those with complete data (for all covariates used in full models, $\mathrm{n}=3759$ ). The same stress-abnormal glucose metabolism relationships were observed in both datasets, with a similar proportion of people who developed abnormal glucose 
metabolism at follow-up and mean values for perceived stress and stressful life events not shown to be appreciably different.

Perceived stress and life events were included individually in the models as the predictor variables. Model 1 included age, gender, and individual-level socioeconomic position (education). Adjustment was made for education to accommodate potential moderation of the relationship between the stressors and abnormal glucose metabolism. Stress has been shown to have a differential impact on health between men and women (13, 17, 19), and therefore we explored gender by stress interactions. The interaction between gender and perceived stress was $p=0.059$, suggesting the need to stratify by gender. Model 2 incorporated the baseline health behaviors (physical activity, smoking [binary variable, current versus ex- and non-smoker], alcohol consumption, and sedentary behavior). Model 3 included adiposity at baseline (waist circumference), and the final model added the components of the metabolic syndrome, systolic blood pressure (SBP - modeled as a continuous variable, per $10 \mathrm{mmHg}$ ), triglycerides (logarithmically transformed), high-density lipoprotein (HDL) cholesterol, and baseline fasting glucose levels. In sensitivity analyses, to examine the relationships between perceived and life events stress and abnormal glucose metabolism more closely, gender-stratified analyses included separately each of the four Perceived Stress Questionnaire components (worries, tension, joy, and demands), as well as the individual life events (all modeled in quartiles), in the same logistic regression models described previously. In addition, we stratified the analyses by the glucose tolerance categories, 'prediabetes' (impaired fasting glucose and impaired glucose tolerance) and type 2 diabetes. Statistical analyses were performed using SPSS 18 and the significance level was set at $p>0.05$.

\section{Results}




\section{Baseline characteristics (table 2)}

In analyses of baseline characteristics stratified by gender, there were significant differences between those people who did and did not develop abnormal glucose metabolism over five years. People who developed abnormal glucose metabolism were older $(p<0.001)$, and more likely to be male $(p=0.002)$. After age-adjustment, men and women with abnormal glucose metabolism at follow-up reported lower levels of education ( $p=0.010$ for men, NS for women). Women who developed abnormal glucose metabolism reported higher levels of perceived stress at baseline $(p=0.010)$; this pattern was not observed among men. In general, those who developed abnormal glucose metabolism had lower levels of physical activity (NS for men, $p=0.021$ for women) and greater central obesity ( $p<0.001$ in both), with less healthy lipid and glucose metabolism profiles. Perceived stress and stressful life events were highly correlated in men and women (Spearman's correlations, $p<0.001, \mathrm{r}=0.41$, and $p<0.001, \mathrm{r}$ $=0.49$ respectively)

\section{Relationship between perceived stress and abnormal glucose metabolism (table 3)}

In women, there was a graded relationship between perceived stress and incident abnormal glucose metabolism after adjustment for age and education $(p=0.005, p=0.003$ for perceived stress as a continuous variable. A curvilinear relationship was tested by including continuous perceived stress as a squared term in the model; this was non-significant, $p=0.79$ ). Women reporting high levels of stress were significantly more likely to develop abnormal glucose metabolism over five years (ORs for the highest and second highest quartiles, respectively, OR 1.68 (1.06-2.66) and OR 1.89 (1.19-2.99)) compared with those in the lowest quartile. With the inclusion of the baseline health behaviors in Model 2, perceived stress remained a significant predictor of abnormal glucose metabolism at follow-up (for the highest two quartiles, OR $1.63(1.03-2.59)$ and OR 1.84 (1.16-2.92)). The relationship 
between perceived stress and abnormal glucose metabolism persisted after adjustment for adiposity in Model 3 (OR 1.69 (1.06-2.47) in the highest quartile). In the final model, with the inclusion of the metabolic syndrome components and baseline fasting glucose, perceived stress remained a significant predictor of abnormal glucose metabolism (OR 1.61 (1.00-2.58) and OR 1.72 (1.07-2.76), in the highest two quartiles of perceived stress respectively).

In men, perceived stress was not a significant risk factor for abnormal glucose metabolism development over the follow-up period in any of the models (OR 1.22 (0.771.94) and OR 1.18 (0.73-1.90) for the highest quartile of perceived stress, in Models 1 and 4, respectively. Perceived stress was tested as a continuous variable, $p=0.46$, and in a curvilinear relationship with abnormal glucose metabolism, $p=0.97$ ).

\section{Sensitivity analyses}

To understand more clearly the aspects of perceived stress relevant to the association with abnormal glucose metabolism, we deconstructed perceived stress into the four factors previously identified within the Perceived Stress Questionnaire (36). The results presented reflect the highest quartile of Perceived Stress Questionnaire component compared with lowest quartile. In women, there was a trend towards worries being a risk factor for abnormal glucose metabolism; however it did not reach significance (OR 2.26 (0.82-6.24)). Tension was shown to be a significant predictor of abnormal glucose metabolism, even after adjustment for covariates (OR 2.15 (1.35-3.44)). The lowest quartile of joy was associated with a significantly increased risk of abnormal glucose metabolism development after adjustment for age and education (OR 1.77 (1.19-2.64)) and health behaviors (OR 1.66 (1.11$2.49)$ ); this relationship was attenuated by the inclusion of adiposity (OR $1.44(0.95-2.17))$. There was no relationship between demands and abnormal glucose metabolism among women in this sample (OR $1.15(0.73-1.82))$. 
Among men, worries and tension did not predict abnormal glucose metabolism development (OR 0.35 (0.05-2.67) and OR 1.13 (0.70-1.83), respectively). Interestingly, low levels of joy increased the risk of incident abnormal glucose metabolism in men (OR 1.56 (1.04-2.35)). The inclusion of health behaviors and adiposity reduced this relationship to non-significance (OR $1.46(0.96-2.20)$ and OR 1.42 (0.93-2.16), respectively). Demands did not predict abnormal glucose metabolism in men (OR $1.13(0.69-1.84))$.

In analyses that divided abnormal glucose metabolism into 'prediabetes' (impaired fasting glucose and impaired glucose tolerance) and type 2 diabetes, the relationship between perceived stress and 'prediabetes' was significant (OR 1.48 (1.08-2.02) for the highest quartile compared with lowest). Perceived stress was not a significant predictor of diabetes incidence, although the magnitude of the OR was very similar to the impact of perceived stress on 'prediabetes'(OR $1.46(0.69-3.10))$.

\section{Relationship between stressful life events and abnormal glucose metabolism (table 4)}

After age- and education-adjustment (Model 1), there was an inconsistent relationship between life events and abnormal glucose metabolism among women; those in the third quartile of stressful life events were the only group at an increased risk of developing abnormal glucose metabolism (OR 1.68 (1.05-2.69)), compared with people in the lowest quartile of stress ( $p=0.056$ for trend with total life events included as a continuous variable, there was no curvilinear relationship between life events and abnormal glucose metabolism, $p$ $=0.83$ ) with no change in this pattern after making additional adjustments in Models 2-4.

In men, stressful life events did not predict incident abnormal glucose metabolism at any stage of analyses (OR $1.30(0.85-2.00)$ and OR $1.20(0.77-1.88)$ for the highest quartile in Models 1 and 4, respectively). 
The individual life stressors were separately examined to understand their relative importance; none were found to be independently related to abnormal glucose metabolism after adjustment in men or women.

\section{Discussion}

This study provides support for the independent role of stress on the development of abnormal glucose metabolism in women, but not in men. Using objective measures of glucose tolerance and population-based data, these analyses found a dose-response relationship, with women reporting increasing levels of perceived stress at progressively elevated risk for the development of abnormal glucose metabolism over the five-year followup period, compared with people reporting low levels of stress. These findings are important for understanding more about contributing psychosocial influences to the increasing burden of diabetes globally.

Variations between women and men in the impact of stress on abnormal glucose metabolism have been observed previously $(9,13,17,19)$. Substantial literature supports our link between stress and diabetes risk among women $(9,12-13,16,18,29)$, with only a small number of longitudinal studies showing a stronger relationship between different stressors and diabetes (mostly self-reported) among men (17, 19-20). A recent study observed that interactions with obesity explained gender differences in the work stress-diabetes association (14). The authors showed that work stress predicted higher risk of developing type 2 diabetes in the obese but not non-obese women, and yet in men, work stress was associated with a lower risk of diabetes incidence in non-obese but not in obese individuals. This is an interesting finding that requires replication; unfortunately, our sample size was not sufficient in each category of weight status to conduct these interaction analyses. In the literature describing the relationship between CHD and similar psychosocial risk, there is evidence that 
women may be more sensitive to certain psychosocial risk factors, including stress reactions, emotional stress reactions, burnout, family relationships and daily hassles/satisfactions, than men (25-26), and our results are broadly supportive of this.

There are many different ways to measure stress (39); this study included data on perceived stress, which assessed stress reactions, as well as the more 'objective' experience of specific stressful events (40). The degree to which 'objective' stressful events influence one's health is largely affected by the appraisal of the stressful situation (perceptions) (41). We were interested in comparing the impact of the perception of stress versus 'objective' stress (life events) on abnormal glucose metabolism. In this study, the development of incident abnormal glucose metabolism was more sensitive to the perception of stress than experience of discrete life events. To explore this further, we examined the separate components of perceived stress to identify the aspects of this scale that were most closely related to glucose metabolism. In women, the three stress reaction components of worries (although not significant), tension, and joy showed an association with abnormal glucose metabolism, whereas, demands, the external assessment of stress (36), did not act as a risk factor. This supports the idea that the internal appraisal and interpretation of the stressor may drive the impact that stress has on our health. Interestingly, joy was the only perceived stress component shown to be associated with abnormal glucose metabolism in men. This could indicate that the aspect of stress influencing the pathways relevant to glucose metabolism in men is the resultant affective response to stress. This is supported by a study examining the relationship between stress, anxiety, and depression on inflammation; in women, markers of stress were significant risk factors for inflammation, while only depression was associated with inflammation in men (24). We are not aware of other studies that have shown this finding in diabetes, and although supported by work that demonstrates a relationship between depressed affect and diabetes in both men and women (42-43), this finding will certainly 
require replication. The investigation of the potential mediating role of one type of stress on the other's relationship with abnormal glucose metabolism was not possible because the two were too highly correlated to be included in the same model.

The previous studies exploring the role of different types of stressors on diabetes risk have demonstrated ambiguous and inconsistent findings (44), with a substantial focus on work-related stress $(9,12-18)$. Kumari et al (17) found that the effort-reward imbalance model of work stress predicted diabetes incidence among male civil servants only. This contrasts with a recent paper from the Whitehall II study that showed a relationship between elevated work strain and diabetes incidence among middle-aged women, but not men (13). Exploring the role of life events, cross-sectional data from the Hoorn Study indicated that people who had experienced stressful life events during the last five years had a 1.6-fold increase of having undiagnosed diabetes (27). This was supported by findings from the longitudinal Healthy Women Study (29), which showed that women who had at least one very severe stressful life event in the preceding six months were more likely to develop the metabolic syndrome over an average 15 year period than those women who reported mild or no stressful events. However, the analysis of the Whitehall II data by Kumari et al. demonstrated no significant relationship between life events and diabetes incidence in men or women (17). The difference in findings between studies may result from methodological inconsistencies as highlighted in a recent review (39). These include samples from select populations $(13,17)$, failure to include objective measures of diabetes $(17,19,45)$, and reliance on cross-sectional data (12). Therefore, with the use of objective glucose tolerance and longitudinal data from a population-based sample, this study provides considerable support to earlier research linking stress to diabetes risk.

The need for an exploration of the potential mechanisms linking stress and diabetes has been highlighted (39). In this study, health behaviors and adiposity had little influence on 
the relationship between stress and abnormal glucose metabolism. The self-report nature of the behavioral data in this study means that the contributions of these variables may be imprecise, which may partly explain their small impact on the stress-glucose metabolism relationship. Although diet information was collected as part of AusDiab, the lower number of participants with complete diet data meant that its role could not be explored in these models. Despite this, it is possible that the inclusion of adiposity would have captured some of the effects attributable to an unhealthy diet and imperfect measurement of the other behaviors. Previous literature exploring possible mediators in the relationship between stress and diabetes has shown inconsistent results. Most studies combined health behaviors with other covariates making it impossible to identify the potential effects attributable to behaviors (17, 28-29). However, studies that specifically explored potential mediators demonstrated results for (20) and against the role of behavioral mediators $(13,27)$. Rod et al. found that, using data from the prospective cohort Copenhagen City Heart Study, perceived stress at baseline predicted smoking, physical activity and alcohol-drinking behavior, as well as diabetes levels at baseline after ten years of follow-up (20). In contrast, data from the Whitehall II Study supported our findings, showing that health behaviors had little impact on relationship between work stress and diabetes incidence (13). Research using objective behavioral data is needed to accurately measure the extent to which health behaviors mediate the stress-diabetes relationship. Given the remaining significant relationship between perceived stress and glucose metabolism even after multiple adjustment with confounding factors, it is likely that there are other unmeasured direct pathophysiological mechanisms through which stress may influence glucose metabolism. Previous research has suggested that an over-stimulation of the hypothalamic-pituitary-adrenal (46) and sympathetic-adrenal axes (47) and increased inflammation (48) may be involved in these pathways. 
Unfortunately, data on cortisol or adrenaline were not available to explore these causal pathways in more depth.

Additional psychosocial data on constructs such as depression and social support were not collected in this study. Depression is a risk factor for incident chronic diseases, in particular CHD (4), and there is evidence (albeit inconsistent (44)) supporting its role in the development of diabetes (49). Previous studies have also demonstrated social support as a buffer for the detrimental impact of stress on the cardiovascular system (50-51). Norburg et al (9) found an interaction between emotional support and work stress on future risk of diabetes; women with strong emotional support appeared to show a reduced risk of diabetes regardless of their stress levels. This study was unable to examine the potential mediating or moderating role of these other psychosocial constructs, nonetheless, the observed relationship between stress and abnormal glucose metabolism implies that coping resources were insufficient to fully compensate for the impact of stress on glucose metabolism.

For these analyses, we used the category of abnormal glucose metabolism as the incident outcome measure instead of diabetes alone. For this, the categories of impaired fasting glucose, impaired glucose tolerance and diabetes were combined, since they share the common pathophysiological mechanisms of insulin resistance and beta cell dysfunction (52). Given the short follow-up period of five years, we chose to use a binary classification system indicating a wide spread of hyperglycemia and disordered carbohydrate metabolism. The sensitivity analyses that compared the impact of stress on 'prediabetes' and type 2 diabetes separately indicated that the magnitude of the OR was very similar in both glucose tolerance categories.

Although this was a national, population-based sample, the initial $37 \%$ response rate for the AusDiab study (30) at baseline means that the sample may not fully represent the Australian population. For follow-up, the response rate was 59\%. However, an additional 
2261 participants (21\% of the total eligible population) completed a telephone self-reported questionnaire on diabetes status at follow-up. Since there was a trend towards poorer health profiles and increased stress levels among non-responders at follow-up, our sample is likely to have represented a 'healthy' cohort; this may have caused an underestimation of the relationship between stress and abnormal glucose metabolism. The incidence of self-report diabetes was similar in those who responded on the telephone and those who attended the follow-up, and therefore any bias from modest follow up rates is unlikely to impact these results. Other limitations should also be considered. It is possible that people exposed to stress might be more likely to be in contact with their healthcare provider and subsequently more likely to be diagnosed with diabetes. However, this could not have influenced our findings, as we measured both diagnosed and undiagnosed abnormal glucose metabolism. The single time-point assessment of stress also precluded the measurement of change in stress exposure. However, this potential misclassification would be expected to only dilute findings, rather than generate spurious positive results. It is also possible that, had the assessment of stress been closer to the outcome measurement, the strength of the relationship would have been stronger. However, clearly the direct and indirect mechanisms through which stress impacts glucose metabolism are gradual and not immediate, and sufficient time is needed to detect causal links. As indicated by the Cronbach's alpha score for the life events scale, the internal consistency for this instrument was modest. This has been found previously with life events scales (53), since discrete objective events are less likely to cooccur than perceptions of stress. Despite the concurrent measurement of the predictors and covariates in this study, we are confident that the results observed do not reflect distress resulting from baseline knowledge of standard risk factors; stratified analyses by baseline obesity and lipid treatment categories (results not shown) demonstrated the same relationships between perceived stress and glucose metabolism across all categories. 
To our knowledge, this is the first study to demonstrate the predictive role of stress on the development of abnormal glucose metabolism in women, in a population-based sample with objective measures of glucose tolerance. Investigating the possible causal mechanisms linking stress and abnormal glucose metabolism will broaden our understanding of the full profile of diabetes risk factors, and should facilitate the development of new approaches for the prevention and management of diabetes. Healthcare professionals should consider perceptions of stress when assessing the full risk factor profile for development of diabetes.

\section{Conflict of Interest}

The authors have no conflict of interest to disclose.

\section{References}

1. Shaw JE, Sicree RA, Zimmet PZ: Global estimates of the prevalence of diabetes for 2010 and 2030. Diabetes Res Clin Pract. 2010, 87:4-14.

2. Magliano DJ, Barr ELM, Zimmet PZ, et al.: Glucose indices, health behaviors, and incidence of diabetes in Australia. Diabetes Care. 2008, 31:267-272.

3. Everson-Rose SA, Lewis TT: Psychosocial factors and cardiovascular diseases. Ann Rev Public Health. 2005, 26:469-500.

4. Rozanski A, Blumenthal JA, Davidson KW, Saab PG, Kubzansky L: The epidemiology, pathophysiology, and management of psychosocial risk factors in cardiac practice: The emerging field of behavioral cardiology. J Am Coll Cardiol. 2005, 45:637-651.

5. Grundy SM, Benjamin IJ, Burke GL, et al.: Diabetes and cardiovascular disease : A statement for healthcare professionals from the American Heart Association. Circulation. 1999, 100:1134-1146. 
6. Eriksson AK, Ekbom A, Granath F, et al.: Psychological distress and risk of prediabetes and Type 2 diabetes in a prospective study of Swedish middle-aged men and women. Diabetic Med. 2008, 25:834-842.

7. Knol M, Twisk J, Beekman A, et al.: Depression as a risk factor for the onset of type 2 diabetes mellitus. A meta-analysis. Diabetologia. 2006, 49:837-845.

8. Mezuk B, Eaton WW, Albrecht S, Golden SH: Depression and type 2 diabetes over the lifespan: a meta-analysis. Diabetes Care. 2008, 31:2383-2390.

9. Norberg M, Stenlund H, Lindahl B, et al.: Work stress and low emotional support is associated with increased risk of future type 2 diabetes in women. Diabetes Res Clin Pract. 2007, 76:368-377.

10. Lazarus R, Folkman S: Stress, appraisal and coping. New York: Springer Publishing, 1984.

11. Puterman E, Adler N, Matthews KA, Epel E: Financial strain and impaired fasting glucose: The moderating role of physical activity in the Coronary Artery Risk Development in Young Adults Study. Psychosom Med. 2012, 74:187-192.

12. Agardh EE, Ahlbom A, Andersson T, et al.: Work stress and low sense of coherence is associated with type 2 diabetes in middle-aged Swedish women. Diabetes Care. 2003, 26:719-724.

13. Heraclides A, Chandola T, Witte DR, Brunner EJ: Psychosocial stress at work doubles the risk of type 2 diabetes in middle-aged women. Diabetes Care. 2009, 32:22302235.

14. Heraclides AM, Chandola T, Witte DR, Brunner EJ: Work stress, obesity and the risk of type 2 diabetes: Gender-specific bidirectional effect in the Whitehall II Study. Obesity. 2011, 20:428-433. 
15. Kawakami N, Araki S, Takatsuka N, Shimizu H, Ishibashi H: Overtime, psychosocial working conditions, and occurrence of non-insulin dependent diabetes mellitus in Japanese men. J Epidemiol Comm Health. 1999, 53:359-363.

16. Kroenke $\mathrm{CH}$, Spiegelman D, Manson J, et al.: Work characteristics and incidence of type 2 diabetes in women. Am J Epidemiol. 2007, 165:175-183.

17. Kumari M, Head J, Marmot M: Prospective study of social and other risk factors for incidence of type 2 diabetes in the Whitehall II study. Arch Intern Med. 2004, 164:18731880.

18. $\mathrm{Xu} \mathrm{W}$, Hang J, Gao W, et al.: Association between effort-reward imbalance and glycosylated hemoglobin (HbA1c) among Chinese workers: results from SHISO study. Int Arch Occup Environ Health. 2012, 85:215-220.

19. Kato M, Noda M, Inoue M, Kadowaki T, Tsugane S: Psychological factors, coffee and risk of diabetes mellitus among middle-aged Japanese: a population-based prospective study in the JPHC study cohort. Endocr J. 2009, 56:459-468.

20. Rod NH, Gronbaek M, Schnohr P, Prescott E, Kristensen TS: Perceived stress as a risk factor for changes in health behaviour and cardiac risk profile: a longitudinal study. $J$ Intern Med. 2009, 266:467-475.

21. Siegrist J, Peter R, Junge A, Cremer P, Seidel D: Low status control, high effort at work and ischemic heart disease: Prospective evidence from blue-collar men. Soc Sci Med. $1990,31: 1127-1134$

22. Landsbergis PA, Schnall PL, Warren K, Pickering TG, Schwartz JE: Association between ambulatory blood pressure and alternative formulations of job strain. Scand $J$ Work Environ Health. 1994, 20:349-363.

23. Cosgrove MP, Sargeant LA, Caleyachetty R, Griffin SJ: Work-related stress and Type 2 diabetes: systematic review and meta-analysis. Occup Med. 2012. 
24. Toker S, Shirom A, Shapira I, Berliner S, Melamed S: The association between burnout, depression, anxiety, and inflammation biomarkers: C-reactive protein and fibrinogen in men and women. J Occup Health Psychol. 2005, 10:344-362.

25. Hallman T, Burell G, Setterlind S, Odén A, Lisspers J: Psychosocial risk factors for coronary heart disease, their importance compared with other risk factors and gender differences in sensitivity. J Cardiovasc Risk. 2001, 8:39-49.

26. Almeida DM: Resilience and vulnerability to daily stressors assessed via diary methods. Curr Dir Psychol Sci. 2005, 14:64-68.

27. Mooy JM, de Vries H, Grootenhuis PA, Bouter LM, Heine RJ: Major stressful life events in relation to prevalence of undetected type 2 diabetes: the Hoorn Study. Diabetes Care. 2000, 23:197-201.

28. Toshihiro M, Saito K, Takikawa S, et al.: Psychosocial factors are independent risk factors for the development of Type 2 diabetes in Japanese workers with impaired fasting glucose and/or impaired glucose tolerance1. Diabetic Med. 2008, 25:1211-1217.

29. Räikkönen K, Matthews KA, Kuller LH: Depressive symptoms and stressful life events predict metabolic syndrome among middle-aged women. Diabetes Care. 2007, $30: 872-877$.

30. Dunstan DW, Zimmet PZ, Welborn TA, et al.: The Australian Diabetes, Obesity and Lifestyle Study (AusDiab)--methods and response rates. Diabetes Res Clin Pract. 2002, 57:119-129.

31. Australian Institute of Health and Welfare: The Active Australia Survey: A Guide and Manual for Implementation, Analysis and Reporting. Canberra: Australian Institute of Health and Welfare, 2003.

32. Australian Institute of Health and Welfare: Standard questions on the use of tobacco among adults. Canberra: Australian Institute of Health and Welfare, 1998. 
33. Ireland P, Jolley D, Giles G, et al.: Development of the Melbourne FFQ: a food frequency questionnaire for use in an Australian prospective study involving an ethnically diverse cohort Asia Pacific J Clin Nutr. 1994, 3:19-31.

34. Dunstan DW, Salmon J, Owen N, et al.: Associations of TV viewing and physical activity with the metabolic syndrome in Australian adults. Diabetologia. 2005, 48:22542261.

35. Levenstein S, Prantera C, Varvo V, et al.: Development of the perceived stress questionnaire: A new tool for psychosomatic research. J Psychosom Res. 1993, 37:19-32.

36. Fliege H, Rose M, Arck P, et al.: The Perceived Stress Questionnaire (PSQ) Reconsidered: Validation and reference values from different clinical and healthy adult samples. Psychosom Med. 2005, 67:78-88.

37. Caldwell JR, Theisen V, Kaunisto CA, et al.: Psychosocial factors influence control of moderate and severe hypertension. Soc Sci Med. 1983, 17:773-782.

38. World Health Organisation: Definition, diagnosis and classification of diabetes mellitus and its complications. Part 1: Diagnosis and classification of diabetes mellitus. Geneva, 1999.

39. Pouwer F, Kupper N, Adriaanse MC: Does emotional stress cause type 2 diabetes mellitus? A review from the European Depression in Diabetes (EDID) Research Consortium. Discovery Med. 2010, 9:112-118.

40. Kanner AD, Coyne JC, Schaefer C, Lazarus RS: Comparison of two modes of stress measurement: Daily hassles and uplifts versus major life events. J Behav Med. 1981, 4:1-39.

41. Cohen S, Kamarck T, Mermelstein R: A global measure of perceived stress. J Health Soc Behav. 1983, 24:385-396.

42. Engum A: The role of depression and anxiety in onset of diabetes in a large population-based study. J Psychosom Res. 2007, 62:31-38. 
43. Kawakami N, Takatsuka N, Shimizu H, Ishibashi H: Depressive symptoms and occurrence of type 2 diabetes among Japanese men. Diabetes Care. 1999, 22:1071-1076.

44. Chida Y, Hamer M: An association of adverse psychosocial factors with diabetes mellitus: a meta-analytic review of longitudinal cohort studies. Diabetologia. 2008, 51:21682178.

45. Melamed S, Shirom A, Toker S, Shapira I: Burnout and risk of type 2 diabetes: a prospective study of apparently healthy employed persons. Psychosom Med. 2006, 68:863869.

46. Björntorp P: Body fat distribution, insulin resistance, and metabolic diseases. Nutrition. 1997, 13:795-803.

47. Lloyd C, Smith J, Weinger K: Stress and diabetes: A review of the links. Diabetes Spectr. 2005, 18:121-127.

48. Dandona P, Aljada A, Bandyopadhyay A: Inflammation: the link between insulin resistance, obesity and diabetes. Trends Immunol. 2004, 25:4-7.

49. Golden SH, Lazo M, Carnethon $\mathrm{M}$, et al.: Examining a bidirectional association between depressive symptoms and diabetes. JAMA. 2008, 299:2751-2759.

50. Christenfeld N, Gerin W, Linden W, et al.: Social support effects on cardiovascular reactivity: is a stranger as effective as a friend? Psychosom Med. 1997, 59:388-398.

51. Grant N, Hamer M, Steptoe A: Social isolation and stress-related cardiovascular, lipid, and cortisol responses. Ann Behav Med. 2009, 37:29-37.

52. Abdul-Ghani MA, Tripathy D, DeFronzo RA: Contributions of $\beta$-cell dysfunction and insulin resistance to the pathogenesis of impaired glucose tolerance and impaired fasting glucose. Diabetes Care. 2006, 29:1130-1139. 
53. Compas B, Slavin L, Wagner B, Vannatta K: Relationship of life events and social support with psychological dysfunction among adolescents. J Youth Adolesc. 1986, 15:205221. 
Table 1: Items included in Perceived Stress Questionnaire and life events scale

Perceived stress items
1. You feel rested
2. You feel that too many demands are being
made on you

3. You are irritable or grouchy

4. You have too many things to do

5. You feel lonely or isolated

6. You find yourself in situations of conflict

7. You feel you're doing things you really like

8. You feel tired

9. You fear you may not manage to attain your goals

10. You feel calm

11. You have too many decisions to make

12. You feel frustrated

13. You are full of energy

14. You feel tense

15. Your problems seem to be piling up

16. You feel you're in a hurry

17. You feel safe and protected

18. You have many worries

19. You are under pressure from other people

20. You feel discouraged

21. You enjoy yourself

22. You are afraid for the future

23. You feel you are doing things because you
Stressful life events

1. Marriage breakup

2. Death of husband / wife or partner

3. Death of a close family member or close friend

4. Disturbing time with husband / wife or partner

5. Disturbing time with children

6. Disturbing time with parents, in-laws or others (not death)

7. Laid off, fired at work, or problem finding a job

8. Trouble with your boss or teacher

9. Trouble at work with a co-worker

10. Felt like you could have, or were having, a nervous breakdown

11. Severe problems with money

12. Sexual difficulties or problems

13. Other troubles not listed 
have to not because you want to

24. You feel criticized or judged

25. You are lighthearted

26. You feel mentally exhausted

27. You have trouble relaxing

28. You feel loaded down with responsibility

29. You have enough time for yourself

30. You feel under pressure from deadlines 
Table 2: Baseline characteristics according to glucose metabolism categories at followup

Category of glucose metabolism

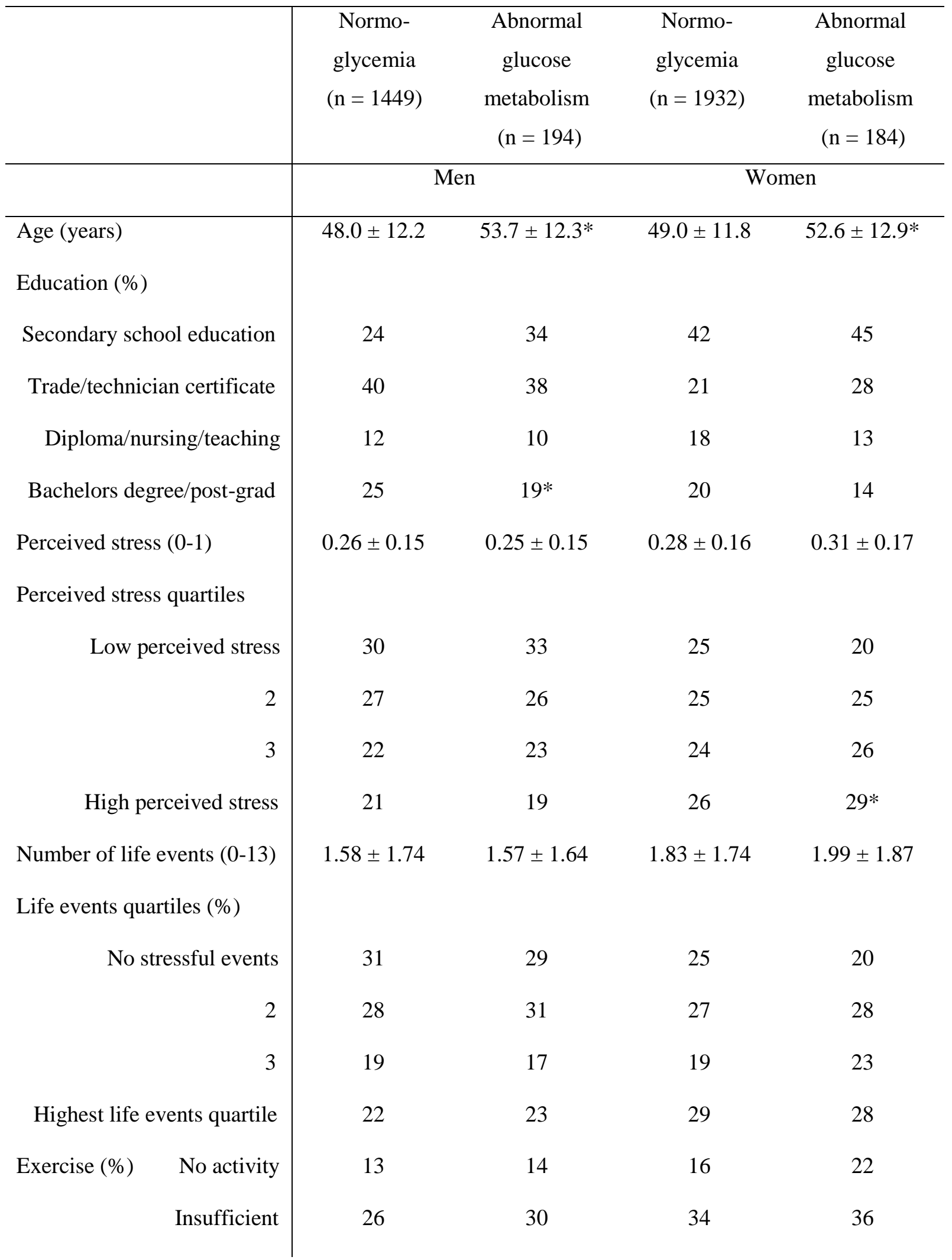


Category of glucose metabolism

\begin{tabular}{|c|c|c|c|c|}
\hline & $\begin{array}{l}\text { Normo- } \\
\text { glycemia } \\
(\mathrm{n}=1449)\end{array}$ & $\begin{array}{c}\text { Abnormal } \\
\text { glucose } \\
\text { metabolism } \\
(\mathrm{n}=194)\end{array}$ & $\begin{array}{l}\text { Normo- } \\
\text { glycemia } \\
(\mathrm{n}=1932)\end{array}$ & $\begin{array}{c}\text { Abnormal } \\
\text { glucose } \\
\text { metabolism } \\
(\mathrm{n}=184)\end{array}$ \\
\hline & \multicolumn{2}{|c|}{ Men } & \multicolumn{2}{|c|}{ Women } \\
\hline $\begin{array}{ll} & \text { Active } \\
\text { Smoking }(\%) & \end{array}$ & 61 & 56 & 50 & $42 *$ \\
\hline Never smoked & 56 & 45 & 65 & 69 \\
\hline Ex-smoker & 31 & 40 & 25 & 24 \\
\hline Current smoker & 13 & $16^{*}$ & 10 & 8 \\
\hline Alcohol intake (grams/day) & $12.8(3.7-28.0)$ & $12.7(2.4-35.1)$ & $3.4(0.5-12.6)$ & $1.38(0.3-10.2)$ \\
\hline Sedentary behavior & $12(7-17)$ & $12(6-19)$ & $10(5-15)$ & $11(5-18)^{*}$ \\
\hline $\begin{array}{l}\text { Diet quality (using Diet } \\
\text { Quality Index) }\end{array}$ & $64.2 \pm 12.5$ & $64.1 \pm 12.9$ & $68.6 \pm 12.0$ & $68.0 \pm 11.9$ \\
\hline Waist circumference $(\mathrm{cm})$ & $94.9 \pm 10.1$ & $100.6 \pm 10.4^{*}$ & $81.9 \pm 11.5$ & $89.8 \pm 13.1^{*}$ \\
\hline Systolic BP (mmHg) & $129 \pm 14$ & $135 \pm 18^{*}$ & $122 \pm 16$ & $130 \pm 20^{*}$ \\
\hline Diastolic BP (mmHg) & $74 \pm 11$ & $76 \pm 11^{*}$ & $65 \pm 10$ & $67 \pm 11$ \\
\hline Total cholesterol (mmol/l) & $5.6 \pm 1.0$ & $5.8 \pm 1.0^{*}$ & $5.5 \pm 1.0$ & $5.9 \pm 1.1^{*}$ \\
\hline HDL cholesterol (mmol/l) & $1.3 \pm 0.3$ & $1.2 \pm 0.3 *$ & $1.6 \pm 0.4$ & $1.5 \pm 0.3^{*}$ \\
\hline Triglycerides (mmol/l) & $1.3(0.9-1.9)$ & $1.5(1.1-2.3)^{*}$ & $1.0(0.7-1.5)$ & $1.4(1.0-1.9)^{*}$ \\
\hline $\begin{array}{l}\text { Fasting plasma glucose } \\
(\mathrm{mmol} / \mathrm{l})\end{array}$ & $5.4 \pm 0.3$ & $5.5 \pm 0.4^{*}$ & $5.1 \pm 0.4$ & $5.4 \pm 0.4^{*}$ \\
\hline $\begin{array}{l}\text { Post-load plasma glucose } \\
(\mathrm{mmol} / \mathrm{l})\end{array}$ & $5.3 \pm 1.1$ & $5.9 \pm 1.2^{*}$ & $5.4 \pm 1.1$ & $6.1 \pm 1.1^{*}$ \\
\hline $\begin{array}{l}\text { Family history of diabetes } \\
\text { (\%) }\end{array}$ & 27 & 26 & 38 & 35 \\
\hline
\end{tabular}

Data are presented as means \pm SD and percentages (\%). Sedentary behavior (television viewing) and triglyceride values presented as medians and interquartile ranges. ${ }^{*} p<0.05$, planned comparisons 
between categories of glucose metabolism using ANCOVAs, logistic regression and Mann-Whitney tests as appropriate, adjusting for age differences. 
Table 3: Adjusted odds ratios (95\% CIs) showing effects of high levels of perceived stress as a predictor of incident abnormal glucose metabolism

\begin{tabular}{|c|c|c|c|c|}
\hline & \multicolumn{4}{|c|}{ Quartiles of stressful life events } \\
\hline & Lowest & 2 & 3 & Highest \\
\hline Men & & & & \\
\hline Adjusted for age and education & 1 & $1.09(0.72-1.64)$ & $1.31(0.85-2.02)$ & $1.22(0.77-1.94)$ \\
\hline $\begin{array}{l}\text { Adjusted for age, education, and } \\
\text { health behaviors }\end{array}$ & 1 & $1.06(0.70-1.60)$ & $1.28(0.83-1.98)$ & $1.17(0.74-1.85)$ \\
\hline $\begin{array}{l}\text { Adjusted for age, education, } \\
\text { health behaviors, and adiposity }\end{array}$ & 1 & $1.07(0.71-1.63)$ & $1.31(0.84-2.03)$ & $1.15(0.72-1.84)$ \\
\hline $\begin{array}{l}\text { Adjusted for age, education, } \\
\text { health behaviors, adiposity, and } \\
\text { metabolic syndrome } \\
\text { components }\end{array}$ & 1 & $1.07(0.70-1.63)$ & $1.34(0.85-2.09)$ & $1.18(0.73-1.90)$ \\
\hline Women & & & & \\
\hline Adjusted for age and education & 1 & $1.42(0.90-2.24)$ & $1.68(1.06-2.66)$ & $1.89(1.19-2.99)$ \\
\hline $\begin{array}{l}\text { Adjusted for age, education, and } \\
\text { health behaviors }\end{array}$ & 1 & $1.41(0.89-2.23)$ & $1.63(1.03-2.59)$ & $1.84(1.16-2.92)$ \\
\hline $\begin{array}{l}\text { Adjusted for age, education, } \\
\text { health behaviors, and adiposity }\end{array}$ & 1 & $1.37(0.86-2.19)$ & $1.55(0.97-2.47)$ & $1.69(1.06-2.71)$ \\
\hline $\begin{array}{l}\text { Adjusted for age, education, } \\
\text { health behaviors, adiposity, and } \\
\text { metabolic syndrome } \\
\text { components }\end{array}$ & 1 & $1.37(0.85-2.19)$ & $1.61(1.00-2.58)$ & $1.72(1.07-2.76)$ \\
\hline
\end{tabular}

Lowest quartile of perceived stress used as reference category. Health behaviors included: physical activity, smoking, alcohol consumption, and sedentary behavior. Metabolic syndrome components included: baseline systolic blood pressure, triglycerides, HDL cholesterol, and fasting blood glucose. 
Table 4: Adjusted odds ratios (95\% CIs) showing effects of high levels of stressful life events as a predictor of incident abnormal glucose metabolism

\begin{tabular}{|c|c|c|c|c|}
\hline & \multicolumn{4}{|c|}{ Quartiles of stressful life events } \\
\hline & Lowest & 2 & 3 & Highest \\
\hline Men & & & & \\
\hline Adjusted for age and education & 1 & $1.21(0.82-1.79)$ & $1.05(0.66-1.67)$ & $1.30(0.85-2.00)$ \\
\hline $\begin{array}{l}\text { Adjusted for age, education, } \\
\text { and health behaviors }\end{array}$ & 1 & $1.24(0.83-1.83)$ & $1.07(0.68-1.70)$ & $1.30(0.84-1.99)$ \\
\hline $\begin{array}{l}\text { Adjusted for age, education, } \\
\text { health behaviors, and adiposity }\end{array}$ & 1 & $1.17(0.79-1.75)$ & $1.03(0.64-1.64)$ & $1.23(0.79-1.90)$ \\
\hline $\begin{array}{l}\text { Adjusted for age, education, } \\
\text { health behaviors, adiposity, and } \\
\text { metabolic syndrome } \\
\text { components }\end{array}$ & 1 & $1.22(0.81-1.83)$ & $1.08(0.68-1.75)$ & $1.20(0.77-1.88)$ \\
\hline Women & & & & \\
\hline Adjusted for age and education & 1 & $1.30(0.84-2.04)$ & $1.68(1.05-2.69)$ & $1.42(0.91-2.23)$ \\
\hline $\begin{array}{l}\text { Adjusted for age, education, } \\
\text { and health behaviors }\end{array}$ & 1 & $1.31(0.84-2.05)$ & $1.67(1.04-2.67)$ & $1.41(0.90-2.22)$ \\
\hline $\begin{array}{l}\text { Adjusted for age, education, } \\
\text { health behaviors, and adiposity }\end{array}$ & 1 & $1.25(0.79-1.97)$ & $1.51(0.94-2.44)$ & $1.27(0.80-2.02)$ \\
\hline $\begin{array}{l}\text { Adjusted for age, education, } \\
\text { health behaviors, adiposity, and } \\
\text { metabolic syndrome } \\
\text { components }\end{array}$ & 1 & $1.26(0.80-1.99)$ & $1.49(0.92-2.42)$ & $1.17(0.73-1.86)$ \\
\hline
\end{tabular}

Lowest quartile of life events used as reference category. Health behaviors included: physical activity, smoking, alcohol consumption, and sedentary behavior. Metabolic syndrome components included: baseline systolic blood pressure, triglycerides, HDL cholesterol, and fasting blood glucose. 